\title{
Inclusión y Juego en la Infancia Temprana
}

\author{
Inclusion and Play in Early Childhood
}

\author{
Francisca Cáceres Zúñiga * \\ Maribel Granada Azcárraga \\ María Pomés Correa \\ Universidad Católica del Maule, Chile
}

\begin{abstract}
El presente artículo propone un acercamiento teórico analítico al concepto de juego para favorecer procesos de inclusión en la infancia temprana en niños con desarrollo típico o aquellos que presentan retrasos en el desarrollo o discapacidad. Se esboza un programa de acompañamiento y desarrollo del juego en edades tempranas, considerando un perfil de fortalezas y debilidades de cada niño, en una intervención multidisciplinaria que integra a la familia y considera los aspectos afectivos y socioemocionales como elementos centrales en el desarrollo de habilidades a partir del juego. Se concluye que la participación en sesiones sistemáticas, con una intensidad ajustada a los requerimientos de cada niño permitiría desarrollar funciones cognitivas, comunicativas y sociales, transitando hacia el logro de habilidades complejas.
\end{abstract}

Descriptores: Juego; Infancia; Derechos del niño; Educación; Chile.

\begin{abstract}
This article proposes an analytical theoretical approach to the concept of play for favoring processes of early childhood inclusion in children with typical development or those with developmental delays or disabilities. It outlines a program of support and development of play at early ages, considering a profile of strengths and weaknesses of each child, in a multidisciplinary intervention that integrates to the family and considers the socio-emotional and affective aspect as central elements in the development of skills from play. It is concluded that the systematic participation in session, with an intensity adjusted to the requirements of each child would develop cognitive, communicative and social moving toward the achievement of complex skills.
\end{abstract}

Keywords: Play; Childhood; Rights of the child; Education; Chile.

\section{Introducción}

El presente trabajo aborda el desarrollo del juego y la inclusión como elementos generadores de igualdad entre niños con y sin discapacidad en la infancia temprana. La relevancia de situar el juego en consonancia con la inclusión se enmarca en la mirada cultural del juego (Huizinga, 1972) que partiendo de un enfoque antropológico cultural se enraíza en el seno de una actividad libre, voluntaria, separada de la vida corriente y ajustada a reglas. En complemento con ello, el concepto de “inclusión” es polisémico y multifacético pues se relaciona con los derechos fundamentales de los grupos en desventaja (UNESCO, 2014), personas en situaciones de deprivación social, discapacidad, retrasos o rezagos del desarrollo, dificultades de lenguaje, entre otros. Este concepto, se basa en un principio de derecho orientado a educación de calidad en un ambiente educativo justo, democrático, que promueva la aceptación, valoración y respeto por las diferencias (Booth y Ainscow, 2011; Echeita, 2013; Echeita y Navarro, 2014). La mirada de inclusión

*Contacto: mfcaceres@ucm.cl

ISSN: 0718-7378

www.rinace.net/rlei/
Recibido: $\quad 04 / 08 / 2017$

$1^{a}$ Evaluación: 19/01/2018

Aceptado: 28/03/2018 
en la infancia temprana, se vincula con una noción amplia de inclusión social referida a una forma de realizar acciones para reducir las limitaciones en la participación. “...tiene que ver con la eliminación de todas las barreras para el juego, el aprendizaje y la participación de todos los niños" (Booth, Ainscow y Kingston, 2007, p. 2). Se concibe como un umbral orientador bajo el cual todas las personas tienen la misma importancia e implica el reconocimiento de las diferencias abordado desde principios más amplios que se enmarcan en políticas sociales y educativas conducidas a crear sociedades más justas (Ainscow et al., 2013; UNESCO, 2016).

Este proceso puede desarrollarse desde que los niños son muy pequeños y comienzan sus primeras interacciones sociales a través del juego. El encuentro con otros en instancias de interacción lúdica permite apreciar las diferencias y la aceptación, aspectos que se reflejan en los tipos de juego desarrollados por niños y niñas, en las estrategias utilizadas para interactuar en el juego y en las formas de resolver y dar continuidad a la actividad lúdica (Zych, Ortega-Ruiz y Sibaja, 2016). Al respecto, para Huizinga (1972) el juego es fundamento y factor de la cultura, "mientras se juega hay movimiento, un ir y venir, un cambio, una seriación, enlace y desenlace” (p. 23). Una vez que se participa del juego, éste queda plasmado en la memoria como recuerdo vivido que puede ser transmitido, replicado o repetido en muchos otros instantes. Es por ello que la identificación y promoción de experiencias de juego, en que todos los niños pueden participar, permite privilegiar espacios de relación socialmente inclusivos (Córdoba, Lara y García, 2017), potenciando tramas culturales que favorezcan la aceptación de la diversidad y el reconocimiento de las diferencias desde las primeras interacciones infantiles.

En este artículo el desarrollo de la inclusión social en la infancia temprana hace referencia a las posibilidades brindadas a través del juego, en tanto competencia social para favorecer la interacción entre niños que presentan características diversas, por tanto, se reconoce la importancia de la estimulación del juego en el entorno social, cultural y educativo para generar posibilidades de participación, aprendizaje y desarrollo de niños con y sin discapacidad. Una perspectiva inclusiva propuesta desde la infancia temprana se relaciona con una educación inicial basada en un enfoque de derecho (ONU, 1959,1989), que se sustenta en el acceso de todos los niños y niñas a la educación y una enseñanza de calidad fundada en los valores y principios de los Derechos del Niño.

En este texto la inclusión se enmarca en los elementos que emergen de la interacción social entre niños y niñas respecto del juego, permitiendo visualizar distintas experiencias de participación en la infancia, en contextos de encuentro lúdico. Ello permite visibilizar las posibilidades que brinda la cultura para percibir, interpretar y responder a distintas situaciones sociales en la interacción lúdica entre pares. El planteamiento de base es favorecer que los niños se sientan socialmente competentes en sus interacciones de juego, manteniendo relaciones sociales positivas que impliquen la inclusión. Resulta pertinente la necesidad de examinar un enfoque comprensivo que aborde en forma conjunta el desarrollo del juego y de distintas formas de inclusión en las interacciones infantiles tempranas. Este artículo discute estrategias de seguimiento, implementación y monitoreo de experiencias de juego tendientes a promover la inclusión, brindando la posibilidad de acompañamiento a niños y niñas pequeños y sus familias, con el fin de provocar respuestas oportunas ante los requerimientos de participación lúdica inclusiva con otros que pudiesen ser excluidos. 


\section{Revisión de la literatura}

\subsection{Necesidades de apoyo en la infancia temprana}

Las etapas que cruza una persona durante su vida, constituyen un continuo que refleja el crecimiento, especialización y maduración que alcanza para desenvolverse de forma exitosa en la interacción social. De estas etapas, el desarrollo en la primera infancia es un aspecto central del desarrollo humano y constituye un indicador decisivo en el grado de éxito de las sociedades (OMS, 2017). Al respecto se debe considerar que todos los seres humanos presentan características y necesidades particulares que los hacen únicos e irrepetibles, por tanto, a pesar de que existe un parámetro de desarrollo esperado para cada edad, éste varía dependiendo de las condiciones biológicas y psicosociales que se conjugan en cada persona (Allen y Cowdery, 2015; Owens, 2016). En esta gran diversidad nos encontraremos con niveles muy heterogéneos, especialmente en los niños, algunos que logran un desarrollo típico para su edad y otros que presentan riesgos, retrasos en el desarrollo. Así, el propósito de la educación debe referirse al reconocimiento de esta diversidad, enfatizando el desarrollo integral de cada persona, cualquiera sea su condición. Para conceptualizar estas diferencias la Clasificación Internacional de Funcionamiento (CIF), a partir de un enfoque biopsicosocial, considera el concepto de discapacidad como "las deficiencias, limitaciones en la actividad o restricciones en la participación" (Giaconi, Nahuelhuala y Pedrero, 2017, p. 184) señalando que el "funcionamiento y la discapacidad de una persona se conciben como una interacción dinámica entre los estados de salud (enfermedades, trastornos, traumas, lesiones, etc.) y factores contextuales" (OMS, 2001, p. 12), por ello, un niño podrá sentirse más o menos discapacitado dependiendo de su relación con las barreras o facilitadores que el entorno provea. En este sentido, son cruciales los programas destinados a los primeros años de vida, en consideración a las etapas de desarrollo, modificabilidad cognitiva y plasticidad que caracteriza a la primera infancia y que permitirían prevenir los retrasos en el desarrollo y atender oportunamente a niños que los presentan. Conjuntamente, la atención oportuna y preventiva se fundamenta en los enfoques de atención temprana centrados en los primeros años de la población infantil. Esta mirada permite considerar las ventanas de oportunidades presentes en la infancia temprana (Aranda, 2011; García, 2014) como etapas que sientan las bases del desarrollo cognitivo, lingüístico, emocional y social, permitiendo a los niños una participación plena y activa en la sociedad (OMS, 2013). Al respecto, la experiencia exitosa de Finlandia nos hace reflexionar sobre la importancia de abordar las problemáticas de la niñez y la juventud desde edades tempranas, previniendo en forma oportuna las dificultades o retrasos en el desarrollo y discapacidad. Ello conlleva a aprovechar al máximo las posibilidades de desarrollo, evitando que las situaciones de rezago puedan agravarse (Sahlberg, 2013).

En este artículo, el énfasis en las necesidades de apoyo en la primera infancia está puesto en las ayudas que requieren algunas personas para satisfacer las demandas del entorno y asegurar procesos de aprendizaje de calidad, evitando o reduciendo las formas de exclusión y discriminación. Al respecto, la atención temprana involucra una serie de apoyos y servicios destinados a niños con retrasos en el desarrollo, riesgos o discapacidad para mejorar el desarrollo personal y de las familias fortaleciendo su inclusión social. Así, hay niños que con las medidas y los recursos habituales les es suficiente, otros niños requieren ayudas menos "usuales" y más específicas para avanzar en los procesos de aprendizaje y participación. Desde una perspectiva ecológica (Bronfenbrenner, 1987), independiente de 
los requerimientos comunes o especializados que necesiten para conseguir las habilidades esperadas en cada etapa, se plantea que los niños de todas las edades son especialmente sensibles a los cuidados de los padres o de los responsables primarios (García, 2014), pues con ellos establecen los vínculos afectivos más estables (Martínez-Priego, Anaya-Hamue y Salgado, 2014; Perpiñan, 2003). En esa interacción las familias pueden crear ambientes estimulantes generando una poderosa influencia en el desarrollo (Mc William, 2016). Así desde los primeros meses de vida, un entorno estimulante incidirá en las habilidades que logren los niños. Por tanto, el desarrollo cognitivo y biológico del lactante dependerá de la calidad de estimulación que reciba de su entorno, siendo determinante en el desarrollo de bienestar y salud (OMS, 2017). En este sentido, se propone la atención temprana vinculada a las actividades cotidianas, rutinarias y lúdicas que desarrollan las familias, de esta forma se generarán oportunidades de aprendizaje en contextos naturales, fortaleciendo el sentimiento de competencia de las familias en la toma de decisiones y en las expectativas que generan en torno a sus hijos.

\subsection{Inclusión en la infancia temprana}

La inclusión, para efectos de este texto, se delimita como un proceso que permite a todos los niños y niñas progresar y avanzar en el aprendizaje y la participación (Booth y Ainscow, 2015; UNESCO, 2015) ello conlleva efectuar las modificaciones necesarias para contar con reales posibilidades de accesibilidad curricular y social, además de la creación de ajustes para propiciar sistemas de participación de las personas en su cultura y en su comunidad. Se intenciona una mirada de respuesta a la diversidad garantizando equidad y calidad educativa, aspectos que van más allá de la superación de la pobreza, situaciones de desventaja o privación social (Booth et al., 2015; Echeita, 2013) para situarse en el reconocimiento de cada persona, permitiendo que se sienta igualmente valorada, que reciba apoyos oportunos a sus requerimientos y que participe plenamente en su entorno y comunidad.

La División para la Infancia Temprana (DEC) y la Asociación Nacional para la Educación de Niños Menores (NAYEC) refuerzan la idea de la Inclusión a través de una declaración de toma de posición conjunta (2009), en la cual valoran la pertenencia, aprendizaje y participación de niños con y/o sin discapacidad y sus familias en las distintas actividades y organizaciones de su comunidad, haciendo énfasis en tres conceptos centrales que definirían la inclusión, a saber: acceso, participación y apoyo. Cuando este proceso se enfoca en niños con discapacidad, menores de ocho años, se denomina inclusión en la Infancia Tempana. Estas instituciones han puesto el énfasis en el mejoramiento de los servicios hacia la infancia, intentando aunar criterios sobre la inclusión y sus significados, formas de implementación, normas, resultados y aspectos centrales para consolidar servicios inclusivos de alta calidad. "La inclusión en la infancia temprana encarna los valores, las normas y los procedimientos en que se basa el derecho de todos los bebés, niños menores y sus familias a participar..." (DEC-NAYEC, 2009, p. 2). Por otro lado, se entiende la discapacidad como un concepto que evoluciona, resultado de la interacción con otros y el entorno, por tanto, todos los niños y niñas deben gozar de los derechos humanos y libertades fundamentales (Giaconi, Nahuelhuala y Pedrero, 2017). Se asume la accesibilidad a los entornos físicos, culturales, económicos, sociales y educacionales como una forma de proteger los derechos y la participación de las personas con discapacidad y sus familias al igual que las de todos los integrantes de la comunidad. Asimismo, UNESCO (2009) recalca la importancia de la educación y la atención temprana en la primera infancia, 
a fin de otorgarles bienestar y brindarles mayores oportunidades de desarrollo al ingresar a la escuela.

La inclusión debe vivenciarse y promoverse en escenarios cotidianos, en lugares y actividades desarrolladas en forma recurrente y que forman parte del contexto natural de vida de niños y niñas en la primera infancia. En estas experiencias de vida cotidiana aflora el "jugar" como concepto central de las actividades en las que se desenvuelven los niños y sus familias, levantándose como un escenario natural, propicio para desarrollar habilidades de inclusión de niños que presentan o no discapacidad. El desarrollo de competencias inclusivas podría proyectarse en base a la disposición a jugar con otro, pues desde el juego con sus propias dinámicas, flexibilidad y regulación, se asumen y acogen las diferencias.

\subsection{El juego y sus características}

El juego se ha constituido en fuente de interés para distintas disciplinas a lo largo de la historia y en especial para la educación, debido al aporte que ha significado en los postulados pedagógicos de grandes pensadores tales como: Freinet, Dewey, Pestalozzi y Montessori, entre otros. Aspecto revelador de la esencia del ser humano, en tanto permite demostrar las particularidades de cada sociedad en su desarrollo artístico, lingüístico, jurídico y poético, entre otros (Huizinga, 1972). Las características del juego lo posicionan como un elemento fundamental en el desarrollo del ser humano y de la cultura, mostrando su vinculación con aspectos cognitivos, afectivos y suprabiológicos. Es una experiencia humana trascendental vinculada con variadas formas de manifestar y vivenciar la cultura. Nos hace más humanos y a la vez se constituye en una experiencia de vida que queda anclada en la memoria. El juego con su gran flexibilidad y múltiples dimensiones posibilita la demostración de afecto, preocupación y cuidado, favoreciendo la expresión de la creatividad y la fantasía. Permite, además, el despliegue de la imaginación, la capacidad de disfrutar junto a otros, la expresión de gustos y preferencias, constituyéndose en una oportunidad para despertar la curiosidad y el asombro a partir de una vivencia satisfactoria. Para Huizinga (1972) el juego es un elemento central de la cultura. Las fiestas sagradas, las celebraciones son formas en las que el juego se manifiesta al interior de la cultura, permitiendo fortalecer las expresiones de una comunidad y vigorizar las manifestaciones grupales.

Algunos aspectos característicos del juego se refieren a la posibilidad de crear y re crear mundos, transitar desde la fantasía a la realidad, en una relación biunívoca que permite a los niños ir y venir experimentando sensaciones, construyendo experiencias y conocimiento a través de sus propias vivencias. Estas experiencias se encuentran enraizadas en la cultura, todo lo que ésta posibilita o por el contrario restringe, permitirá caracterizar las formas de juego que desarrollan los niños. En este sentido, el vínculo con pares o con adultos dispuestos a involucrarse en esta experiencia lúdica, se convierte en un elemento estimulante para el desarrollo del juego (Garvey, 1985). Al respecto y siguiendo a Winnicott (2012) en sus sesiones de observación de juego infantil en consultas psicológicas, se puede mencionar que "los chicos juegan con mayor facilidad cuando la otra persona puede y sabe ser juguetona” (p. 88) lo que implica la existencia de un entorno favorecedor del desarrollo del juego y adultos que se incorporen en rutinas y hábitos para propiciar su estimulación.

En el caso de los niños, el juego, otorga la posibilidad de repetir en forma interminable lo que resulta grato y satisfactorio. Además, revela los esfuerzos que cada niño o niña realiza por interpretar, aproximarse y comprender el mundo (Whitebread et al., 2012). Es una 
actividad voluntaria, desinteresada, espontánea y agradable (Caillois, 1938, 1986). Cuando los niños juegan están en alguna forma re-presentando el mundo, haciendo uso de su imaginación para imitar las actuaciones que vivencian en la realidad. Al ser una actividad desinteresada, que no exige la elaboración de un producto determinado ni persigue obtener ganancias, sino, por el contrario, una acción gratuita que se vincula con la fantasía y la distracción, permite que todos puedan desarrollarlo.

\subsection{El juego en la infancia temprana en niños con y sin discapacidad}

La niñez y la infancia temprana son etapas en las que el juego se manifiesta como expresión natural del desarrollo de los niños. Este período es especialmente valioso para el desarrollo y manifestación de interacciones lúdicas, pues el niño es un explorador que va descubriendo infinitas oportunidades para crear, representar el mundo y compartir socialmente. Desde una perspectiva integral y según la convención de derechos del niño especificado en el artículo 31, el juego es un derecho de los niños. Les permite encontrarse en una actividad placentera, demostrando sentimientos de agrado a través de una acción libre que se desarrolla intersubjetivamente, en tanto relación e interacción con otros.

En la infancia temprana, niños y niñas, expresan a partir del desarrollo de conductas psicomotrices, la exploración del mundo, iniciaciones en el lenguaje o las formas de manipular, asir o coger objetos (Owens, 2016). El juego está imbricado en este desarrollo e involucra miradas, trayectorias, recorridos, movimientos, desplazamientos y ademanes que llenan de sentido la acción de los niños. Tal como mencionan Booth, Ainscow y Kingston (2007) “...el juego es importante para el desarrollo a cualquier edad, valioso en sí mismo y no sólo como algo que puede ser controlado y evaluado” (p. 2).

En consecuencia, el juego en la infancia temprana se visualiza como un aspecto esencial para el desarrollo de distintas funciones afectivas, cognitivas y lingüísticas (Owens, 2016). Es una experiencia humana importante que permite desplegar habilidades para representar y explorar el mundo. En la interacción y vinculación lúdica con adultos y pares, el niño adquiere habilidades sociales y potencia sus capacidades afectivas y cognitivas. $\mathrm{Al}$ jugar, interactúa, se conecta y se relaciona con otros, en una situación de placer. "El juego ofrece beneficios para el desarrollo cognitivo, emocional, físico y moral" (American Academy of Pediatrics, 2006; Elkind, 2007, citados en Bay Area Early Childhood Funders, 2007)

Al respecto, la discusión ha comenzado a transitar en relación a las etapas de desarrollo de los niños y su relación con el juego, las habilidades alcanzadas y lo que ocurre con aquellos niños que presentan discapacidad o retrasos en el desarrollo. Las habilidades desplegadas por los niños al jugar, se pueden organizar en secuencias temporales específicas de cada etapa de desarrollo. Estas secuencias serían reveladoras del período lúdico en el que se encuentran niños y niñas, existiendo diferencias entre el desempeño de niños con discapacidad y el desempeño de niños con desarrollo típico.

En este sentido, para los niños con desarrollo típico el juego es una habilidad muy natural que vivencian en forma cotidiana, desarrollándose en los distintos ambientes y situaciones en las que participan. Logran conectarse con otros, utilizar juguetes y objetos para simular situaciones o proyectar eventos, utilizan la imaginación y la creatividad para resolver problemas o encontrar soluciones a sus inquietudes y disfrutan en un involucramiento sostenido que los mantiene motivados por largo tiempo. En el caso de niños con discapacidad, se presentan variaciones en el desarrollo de los distintos aspectos del juego. 
Algunos niños con discapacidad, dependiendo del nivel de desarrollo que hayan alcanzado, muestran descensos en las habilidades de juego, en muchas ocasiones, las habilidades lúdicas no se desarrollan de forma espontánea, se presentan de manera menos regular y en ocasiones con descenso importante respecto de los tipos de juego, las interacciones desarrolladas en tiempos de juego y menor diversificación en el desarrollo de juego simbólico. Según Barton (2015), los niños con discapacidad juegan menos que los niños con desarrollo típico y su juego simbólico es menos variado que el experimentado por los niños con desarrollo típico.

En general, carencias en las habilidades de juego son una manifestación de algún rezago o retraso en el desarrollo. Por ejemplo, niños con discapacidad intelectual presentan retrasos en el desarrollo del juego y del ocio (Quiroga, 1989; Sainz y Mayor, 1989, citados en Peredo, 2016). El juego se caracteriza por demostrar un menor repertorio de habilidades, un lenguaje reducido, una actividad lúdica menos sofisticada, una selección más limitada de materiales y con menores representaciones. Niños con discapacidad severa muestran conductas estereotipadas. Por su parte, niños con discapacidad motora demuestran menor participación debido a dificultades de manipulación y movilidad. Niños con discapacidad visual exhiben menor cantidad de juegos de intercambio social y más tiempo de juego solitario que niños con desarrollo típico. Niños con dificultades de lenguaje muestran menor contacto social y tienen un juego menos organizado.

Las investigaciones señalan que los niños con Trastorno específico de lenguaje desarrollan menos juego simbólico, éstos presentan menor duración y aparecen con menor frecuencia. Utilizan menos materiales y sus juegos se presentan poco creativos (Brown et al., 1975; Lovell, Hoyle y Siddall, 1968; Williams, 1978, citados en Barrachina et al., 2015). Al respecto, las investigaciones en habilidades cognitivas no lingüísticas en niños con trastorno específico de lenguaje (TEL) señalan que niños con TEL presentan una coordinación motora más pobre en términos de habilidades sensoriomotrices (Johston et al., 1981; Robinson, 1987; Stark y Tallal, 1981; Vargha-Khademte et al., 1995, citados en Barrachina et al., 2015). Por otra parte, los niños con déficit atencional manifiestan un juego menos simbólico o social, mostrando frecuentemente actividades de movimientos repetitivos. De esta manera, es posible determinar que ninguna discapacidad podrá ser diagnosticada debido a déficit en las habilidades lúdicas, pero discapacidades específicas sin lugar a dudas, tienen impacto en el tipo de juego desplegado (Garfinkle, 2004). El cuadro 1 muestra una clasificación de los tipos de juego desarrollados por los niños.

Esta clasificación contemporánea permite situar al niño en los tipos de juego a los que accede, integrando las experiencias de juego con otros dominios cognitivos que va desarrollando. Al respecto, Clemente (2006) releva el juego simbólico y su relación con el lenguaje, señalando "el juego simbólico permite fácilmente y a partir de los dos años y medio, introducir verbalmente al niño en discursos descentrados del aquí y el ahora” (p. 22). Así mismo, destaca la posibilidad de adquirir los tiempos verbales cuando los niños se introducen en un mundo narrado, pequeñas historias, personajes y argumentos.

Tal como se ha referido en este artículo, el juego es dinámico y multifacético, pues cambia constantemente sin usar una forma específica ni conductas determinadas al manifestarse. Tampoco es desarrollado en un contexto único ni asociado a un específico conjunto de habilidades. Difícilmente se define por lo que puede considerarse que es a la vez, una acción, un proceso y el producto de la conducta infantil (Sherratt y Peter, 2002).

Cuadro 1. Clasificación de juego contemporáneo 


\begin{tabular}{|c|c|}
\hline $\begin{array}{c}\text { CATEGORÍA DE } \\
\text { JUEGO }\end{array}$ & DESCRIPCIÓN \\
\hline $\begin{array}{l}\text { Juego } \\
\text { sensoriomotor }\end{array}$ & $\begin{array}{l}\text { Explorar el mundo actuando en él a través del tacto, pronunciando, morder, } \\
\text { oler, gustar, golpear, patear. }\end{array}$ \\
\hline Juego relacional & $\begin{array}{l}\text { La exploración de la relación entre las propiedades de los objetos } \\
\text { seleccionados insertando, construcción, agrupación o asociación de diferentes } \\
\text { maneras. }\end{array}$ \\
\hline Juego funcional & $\begin{array}{l}\text { Ganando placer de usar objetos en la forma en que se supone que se utilizará, } \\
\text { como hacer un perro de la corteza juguete, fingiendo beber de una taza. }\end{array}$ \\
\hline Juego simbólico & $\begin{array}{l}\text { Mediante la sustitución de los objetos, atribuyéndole propiedades falsas a un } \\
\text { objeto; imaginando un objeto; o, de manera consciente actuando } \\
\text { interacciones sociales de una secuencia de acciones que por una simple } \\
\text { historia basada en hecho cotidiano. }\end{array}$ \\
\hline $\begin{array}{l}\text { Temática de } \\
\text { fantasía }\end{array}$ & $\begin{array}{l}\text { Pretensión altamente imaginativo y creativo dentro de una narrativa } \\
\text { dinámica. }\end{array}$ \\
\hline
\end{tabular}

Fuente: Recuperado de Sherratt y Peter (2002).

Los autores han optado por describir sus características, indicando que la actividad lúdica es voluntaria, flexible, simbólica, significativa, gobernada por reglas, placentera y episódica, activa y no literal entre otras (p. ej., Fromberg, 2002; Hughes, 1991; Smith y Vollstedt, 1985). Describir las características del juego a través de la generación de taxonomías, permite conocerlo con mayor especificidad y facilita su evaluación. Las taxonomías admiten la generación de un sistema de clasificación donde cada categoría puede ser definida a través de conductas específicas observables y discretas, haciendo posible la evaluación del juego. Una de las clasificaciones del juego es la propuesta por Parten citado en Espinoza (1999), en la cual se enfatiza el carácter social de la actividad lúdica producida por los niños, tal como se señala en el cuadro 2.

Cuadro 2. Taxonomía del juego social

\begin{tabular}{ll}
\hline \multicolumn{1}{c}{ CATEGORÍA } & \multicolumn{1}{c}{ DEFINICIÓN } \\
\hline Conducta no ocupada & $\begin{array}{l}\text { El niño no juega, pero está ocupado mirando cosas de interés } \\
\text { momentáneo }\end{array}$ \\
\hline Juego solitario & El niño juega solo y con diferentes juguetes cercano a otros niños \\
\hline Espectador & $\begin{array}{l}\text { El niño mira a otros niños jugar, de pie o sentado a una distancia } \\
\text { óptima para hablar, frecuentemente conversa con los niños a los que } \\
\text { está mirando }\end{array}$ \\
\hline Actividad paralela & $\begin{array}{l}\text { El niño juega independientemente, pero con juguetes que lo llevarán } \\
\text { a acercarse a otros niños; el niño juega a lado, pero no con otros } \\
\text { niños. }\end{array}$ \\
\hline $\begin{array}{l}\text { Juego asociativo } \\
\text { Juego suplementario } \\
\text { cooperativo u } \\
\text { arganizado }\end{array}$ & $\begin{array}{l}\text { Uno o dos nintercambian materiales y conversación sobre una } \\
\text { para algunos propósitos, necesitando una división de la tarea y la } \\
\text { organización de los roles. Los niños pertenecen o no al grupo. }\end{array}$ \\
\hline Fuente: Elaboración propia a partir de Espinoza (1999).
\end{tabular}

Los niños pequeños pasan gran cantidad de tiempo jugando, esto ha sido observado en todas las culturas y épocas. Los motivos del juego en la infancia también han sido largamente estudiados y existen diversas teorías que explican la razón de la actividad lúdica, entre ellas el juego utilizado como sobrante de energía, para recreación o como práctica para desarrollar los roles de adultos, entre otros. En este sentido, es posible evaluar la actividad lúdica a través de la observación de conductas de juego y proyectarla como una habilidad que debe estar presente en el currículo infantil para niños con y sin 
discapacidad, constituyéndose en una estrategia de estimulación, aprendizaje e inclusión social.

La propuesta consiste en utilizar estrategias que favorezcan el desarrollo de juegos más variados. Un repertorio de juego simbólico que incluya la enseñanza explícita y sistemática de habilidades lúdicas que permitan a niños con rezago o discapacidad acceder a la dinámica de juego. Una vez que están involucrados en el juego o que los niños han ingresado en esta esfera lúdica, no se interfiere en la dinámica propia del juego, permitiendo que éste se desarrolle con toda naturalidad, dejando al niño lo más libre posible.

\section{Propuesta de abordaje del juego en la infancia temprana}

Es posible identificar diferencias en los tipos de juego practicados por niños con alguna discapacidad en comparación con sus pares de la misma edad sin discapacidad, por esta razón, es importante contar con estrategias de evaluación del juego y con metodologías que permitan incrementar las habilidades lúdicas de niños con retraso en el desarrollo o discapacidad. Desde una perspectiva ecológica (Bronfenbrenner, 1987), se plantea que el juego es el contexto donde se implementan intervenciones, es además una habilidad funcional con un valor práctico, que incrementa oportunidades para actividades normativas. En este sentido, para que un niño logre jugar, debe primero sentirse significativamente atraído y conectado en la situación de juego. Necesita estar participando con materiales y con otros de manera ajustada y con sentido, de esta forma, el juego será una experiencia gratificante y enriquecedora. En este sentido se proponen estrategias diversificadas de enseñanza, dentro de ellas podría considerarse las propuestas del diseño universal de aprendizaje (Meyer, Rose y Gordon, 2014), contemplando variadas opciones de compromiso en el desarrollo del juego, proporcionar múltiples opciones de representación de las actividades lúdicas y favorecer distintas formas de acción y expresión en el desarrollo del juego.

Por otra parte, al considerar la complejidad de la actividad lúdica, hay que visualizar que puede manifestarse en una amplia gama de conductas. Cuando la actividad lúdica desplegada por un niño es simple, el juego con objetos y el juego simbólico se presentan lejanos y distintos, pero en la medida en que el juego se vuelve más avanzado y complejo, la actividad concreta y la actividad simbólica comienzan a fundirse. Lo mismo ocurre al analizar el juego según el desarrollo de habilidades sociales implícitas en él. La actividad lúdica entonces transita desde el juego solitario hasta conseguir uno más cooperativo. Por lo tanto, un juego sofisticado, implica la integración de distintos tipos de juego, desde lo social pero también desde la utilización de objetos y materiales.

La importancia de la estimulación explícita de desarrollo del juego en la infancia temprana reside en potenciar distintas áreas cognitivas y sociales, funcionando como aporte y soporte del desarrollo de los niños, complementando las experiencias lúdicas con las habilidades y áreas que se encuentran en desarrollo. Así Clemente (2006) plantea favorecer el desarrollo del lenguaje utilizando experiencias intencionadas de juego. Sugiere jugar con el niño, desarrollando una propuesta programada y organizada. Recomienda utilizar representaciones humanas habituales en los juguetes de los niños, planificar los escenarios 
y objetos que permitirán la acción e incorporar roles a los muñecos o personajes, imitando las verbalizaciones producidas en forma espontánea por los niños en momentos de juego.

Otra mirada de estimulación en la infancia temprana a partir del juego la propone el Consejo Interdisciplinario de Trastornos del Desarrollo y el Aprendizaje (ICDL) promoviendo un trabajo interdisciplinar para abordar las dificultades de comunicación, relación o pensamiento a partir del Modelo de DIR/Floortime, en la atención de los niños que presentan retrasos o trastornos del desarrollo (Greenspan y Wieder, 1999; Wieder y Greenspan, 2005). Este Modelo considera las etapas de desarrollo (D) en que se encuentra cada niño, las diferencias individuales (I) y las relaciones (R) e Interacciones o los patrones relacionales que afectan las interacciones en edades tempranas. El enfoque plantea un perfil único de cada niño a partir de sus fortalezas y debilidades, integrándolo en un plan de apoyo orientado a que cada niño alcance su máximo potencial. El modelo se basa en el logro de habilidades funcionales que permitan integrar las distintas áreas del desarrollo e interactuar con un propósito y se caracteriza por plantear el rol del afecto y las emociones como aspectos centrales en el logro de habilidades. El área afectiva o socio emocional guía y orienta el desarrollo de las otras áreas cognitivas, otorgando significado a las conductas sociales y comunicativas. Esta propuesta requiere de trabajo multidisciplinario y la participación activa de la familia a fin de comprender este perfil único y desarrollar conductas comunicativas y socioemocionales complejas.

La estrategia Floortime o Juego Circular propone usar el juego de forma sistemática para generar oportunidades de desarrollo y promover círculos de comunicación. Padres o cuidadores primarios participan de sesiones diarias de interacción lúdica que permiten al niño expresar ideas, necesidades y emociones tendiendo puentes entre esta comunicación expresada a través del lenguaje o el juego y la capacidad de organizar experiencias, actos o secuencias en respuestas comunicativas de mayor complejidad (Breinbauer, 2006).

La propuesta de este artículo reside en considerar la utilización explícita de patrones secuenciados de juego, incorporando la estimulación propicia para favorecer el desarrollo autónomo del juego en aquellos niños que presentan juego poco variado, con escaso dinamismo o que no inician interacciones lúdicas. Este proceso involucra parcializar las rutinas de juego, entregando refuerzo oportuno y constante frente a las iniciativas de interacción lúdica, por pequeñas que éstas sean. Aquellos niños que presentan discapacidad o se encuentran en riesgo de presentar rezago o retrasos en su desarrollo se beneficiarían de entornos estimulantes y rutinas de juego sistemáticas e integrales, dando paso a la adquisición de hábitos lúdicos, en secuencias de complejidad creciente, con actividades programadas y estructuradas para ello. Las consideraciones de una propuesta de estimulación temprana, centrada en la familia y en un trabajo multidisciplinario, se basan en respetar los aspectos propios del desarrollo de cada niño, teniendo como referencia el desarrollo típico descrito en la literatura, no obstante, considerando las barreras contextuales y respetando la individualidad, como asimismo el grado de compromiso o requerimientos de apoyo que presenta cada persona. Para ello se han considerado los siguientes aspectos centrales en una Propuesta Integral de Estimulación del Juego: 


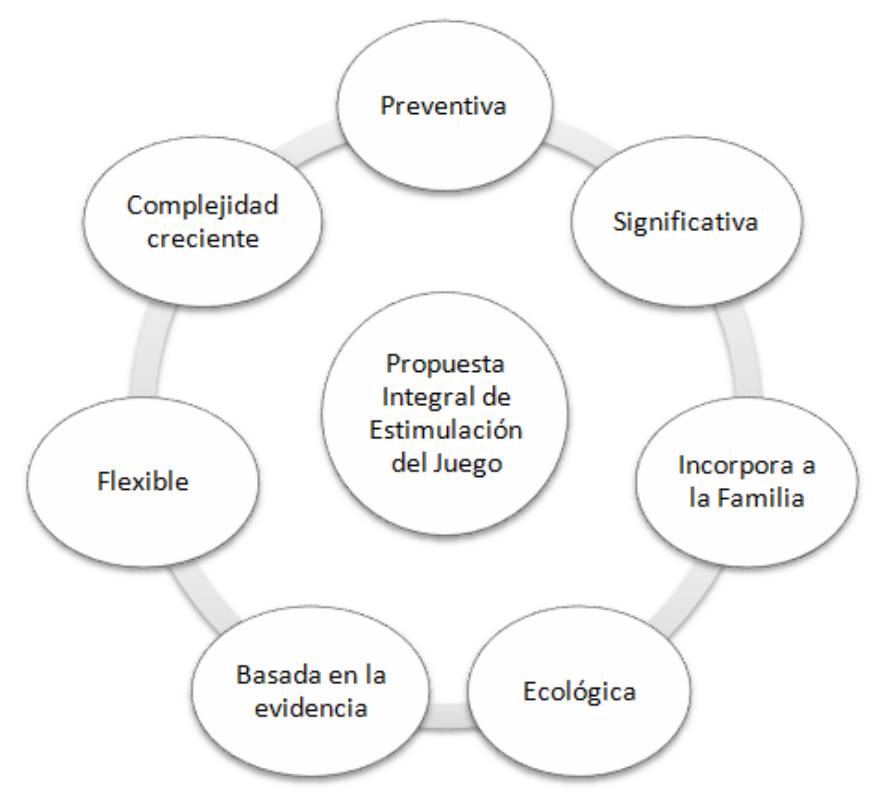

Figura 1. Diagrama de aspectos centrales en la elaboración de una propuesta integral de desarrollo del juego para niños con desarrollo típico, retrasos en el desarrollo y discapacidad

Fuente: Elaboración propia.

Propuesta preventiva: Consiste en generar oportunidades y experiencias de estimulación enriquecidas y variadas de juego infantil para todos los niños. El objetivo es favorecer procesos de interacción, desarrollo cognitivo, emocional y social a partir de los primeros años de vida, considerando la infancia temprana como una etapa central y propicia para el desarrollo del juego.

Propuesta significativa: La estimulación debe enfocarse en proveer situaciones de juego estimulantes, que aborden los intereses de los niños de acuerdo a la etapa del desarrollo en la cual se encuentran. Debe enfocarse en una mirada integral que contemple la estimulación de diferentes áreas cognitivas, estableciendo puentes hacia otras esferas de la vida cotidiana e incrementando la posibilidad de desarrollar juegos en forma independiente. De esta forma, y a partir de un juego que en algunos casos puede ser más dirigido, lograr la trascendencia y proyección a experiencias lúdicas de carácter autónomo.

Propuesta ecológica: Desarrollada en contextos naturales y contextualizados. Ello implica considerar las actividades cotidianas desempeñadas por el niño y las acciones habituales desarrolladas por la familia, como contextos próximos, cercanos y reales a partir de los cuales se planifica el programa de estimulación.

Desarrollada en complejidad creciente: Permite desarrollar habilidades de juego en forma progresiva y debe estar focalizada en el perfil de cada niño. Las pautas secuenciadas y parcializadas, permiten profundizar en rutinas de juego dirigidas, haciéndolas reiterativas, intensas y sistemáticas. De esta forma se brinda la oportunidad de repetir patrones de juego, que, al ser asumidos en forma habitual, comenzarán a integrarse paulatinamente en el proceso de juego espontáneo y autónomo.

Basada en la Evidencia: La propuesta debe contemplar las experiencias exitosas, investigaciones y sistematizaciones en torno al tema. Esto permite fundamentar las 
decisiones de estimulación, considerando los avances, actualizaciones y resultados de las últimas investigaciones, así como literatura especializada en el área de Educación Especial e Infancia Temprana.

Propuesta Flexible: Es importante identificar las posibilidades de acción, el nivel alcanzado por el niño y sus necesidades. Esta línea de base, permite iniciar el programa de estimulación de acuerdo a las habilidades o requerimientos de apoyo, pudiendo pasar sin dificultad de una actividad a otra y comenzar la estimulación en cualquier punto de la secuencia propuesta. Este enfoque contempla la participación de niños que presentan buen nivel de desarrollo psicomotor o buenas habilidades de juego, así como también a aquellos que presentan retrasos en el desarrollo o discapacidad.

Incorporación de la familia: El trabajo con la familia, permite identificar las posibilidades de acción, expectativas, intereses, requerimientos y objetivos prioritarios que contemplan los padres o personas involucradas frecuentemente en las interacciones con el niño. Ello implica desarrollar en forma colaborativa, acciones de planificación, monitoreo y retroalimentación de las actividades y objetivos logrados en cada fase de aplicación del plan de estimulación. En este proceso se considera relevante el modelamiento y mediación del profesional responsable de guiar el acompañamiento de rutinas específicas de juego.

La secuencia de estimulación de juego basada en una propuesta en complejidad creciente se presenta en la figura 2. El diagrama explica un plan de estimulación con un enfoque de complejidad creciente. Comienza por la propuesta de interacción inicial en juego conjunto entre el adulto y el niño, sin embargo, dependiendo del perfil de cada niño, es posible partir desde cualquier punto del plan propuesto.

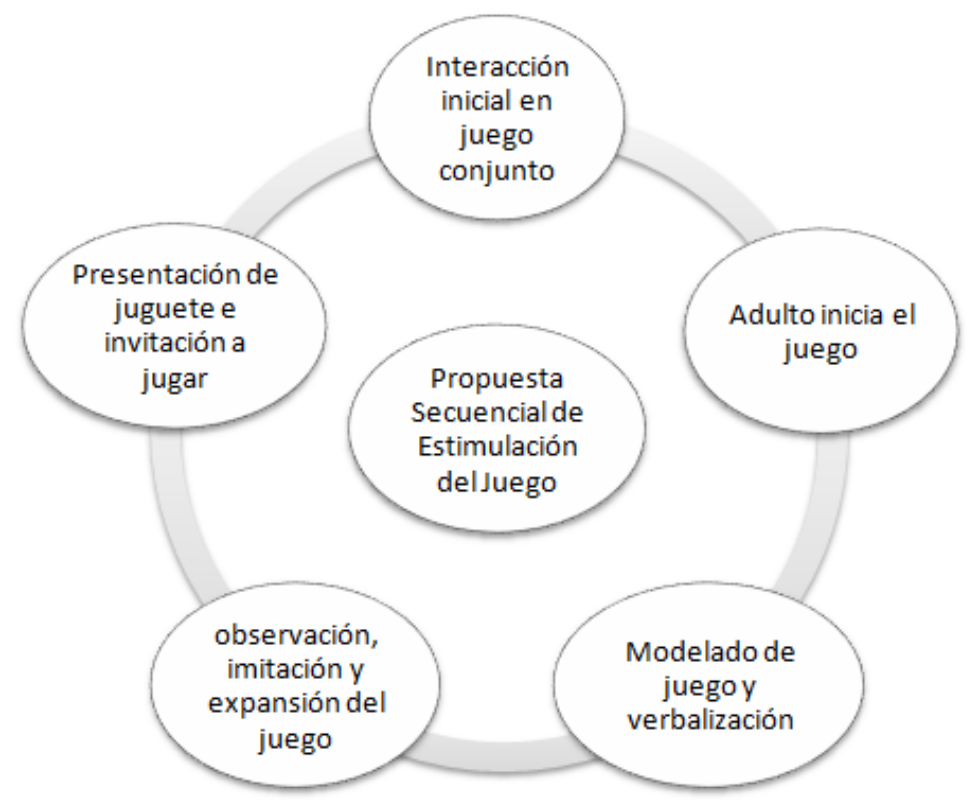

Figura 2. Plan secuencial de estimulación del juego

Fuente: Elaboración propia.

Interacción inicial de juego conjunto: El adulto y el niño se sientan en el suelo con variados juguetes, generando la posibilidad de inicio de juego por parte del niño (cuadro 3). 
El adulto inicia una actividad de juego en la cual involucra corporalmente al niño y verbalización: Si el niño no inicia el juego en forma independiente, el acompañante inicia una actividad simple de juego en la cual implica corporalmente al niño y describe la acción que va realizando.

\section{Ej.1: El adulto toma la mano del niño y mueve un auto de juguete y habla en forma simultánea describiendo lo realizado. \\ Ej.2: El adulto toma los brazos de la niña y mecen una muñeca, verbaliza en forma simultánea las acciones.}

Modelado de juego y verbalización: El adulto realiza alguna actividad de juego para intencionar la imitación por parte del niño, muestra una actividad de juego.

Ej.1: El adulto lanza una pelota a una red y describe simultáneamente lo que está realizando.

Ej.2: El adulto sopla un globo desplazándolo con el aire de su boca y comenta con habla paralela todas las acciones que realiza.

Observación, imitación y expansión del juego: El adulto observa la actitud del niño frente al modelado anterior y ante cualquier acción de iniciar un juego, imita inmediatamente la conducta del niño, verbaliza lo realizado y amplia el patrón de juego comenzado por el niño.

Ej.1: El niño toma el auto de juguete. Inmediatamente el adulto toma el auto de juguete y lo desplaza hacia adelante, hacia atrás y lo guarda en el estacionamiento. Paralelamente relata la acción que va realizando.

Presentar el juguete e invitarlo a jugar: El adulto muestra un juguete, se lo entrega al niño y lo invita explícitamente a jugar. Deja que el niño desarrolle libremente su juego. Otorga verbalizaciones positivas y refuerzo ante las iniciativas del niño.

Ej.1: el adulto muestra una pelota, la hace rodar pasándosela al niño y describe las características de la pelota y del juego, invitando al niño a jugar. Ante la respuesta del niño, entrega elogios. ¡Muy bien!, ¡Me encanta jugar contigo!

El acompañamiento en actividades de juego debe estructurarse en una secuencia progresiva de gran intensidad en el apoyo a moderadas y pequeñas ayudas. Se postula gran acompañamiento a niños que presentan mayor compromiso sensorial, cognitivo, conductual o psicomotriz, el cual se va graduando dependiendo de las características, necesidades y evolución de los niños en sus habilidades de juego.

El desarrollo de estas fases de juego infantil puede considerarse para la estimulación de niños con desarrollo típico o niños que presentan riesgos de retrasos o discapacidad. Permite a partir de la enseñanza explícita de habilidades de juego, intencionar y favorecer lo que otros niños realizan naturalmente, transfiriendo a su entorno natural las experiencias de juego desarrolladas en forma dirigida. Especialmente en el caso de niños con discapacidad otorga posibilidades de mayor participación social e interacción en actividades recreativas. 
Cuadro 3. Ejemplo de guion de estimulación de juego

\begin{tabular}{|c|c|c|}
\hline MOMENTOS DE DESARROLLO DEL JUEGO & GUION SUGERIDO & EJEMPLOS DE ACCIONES \\
\hline Interacción inicial de juego conjunto & $\begin{array}{l}\text { 1.- Explicitación inicial: Hoy vamos a jugar con este auto. } \\
\text { Pregunta e incitación dirigida a respuesta. ¿Con qué vamos a } \\
\text { jugar? Repitámoslo juntos. Acción de juego. }\end{array}$ & $\begin{array}{l}\text { a) Se muestra el auto y se entrega la consigna, } \\
\text { Hoy vamos a jugar con este auto. Luego se } \\
\text { pregunta: ¿con qué vamos a jugar? } \\
\text { b) Repitámoslo juntos: Vamos a jugar con este } \\
\text { auto. } \\
\text { c) Se hace rodar el auto por una pista en varias } \\
\text { oportunidades y se verbaliza. }\end{array}$ \\
\hline $\begin{array}{l}\text { Realización de actividad de juego por parte } \\
\text { del adulto en la cual involucra corporalmente } \\
\text { al niño y verbalización }\end{array}$ & 1.- Ahora vamos a hacer rodar el auto. Hagámoslo avanzar. & $\begin{array}{l}\text { a) Mira yo lo haré primero. Estoy haciendo rodar } \\
\text { el auto (hacer rodar el auto por la pista). } \\
\text { b) Ahora hagámoslo juntos. El adulto toma la } \\
\text { mano del niño y realizan la acción. Estamos } \\
\text { haciendo avanzar al auto. }\end{array}$ \\
\hline Modelado de juego y verbalización & $\begin{array}{l}\text { 3.- Ahora haremos sonar el motor y avanzar el auto por la } \\
\text { pista. Entrega de Elogio. }\end{array}$ & $\begin{array}{l}\text { a) Mira yo lo haré primero. Estoy haciendo sonar } \\
\text { el motor y avanzar el auto (realizar las acciones) } \\
\text { b) ahora hazlo tú. Se le pasa el auto al niño } \\
\text { c) ¡Muy bien! el auto pasea por la pista. }\end{array}$ \\
\hline Observación, imitación y expansión del juego & 4.- Ahora te voy a pasar el auto. & $\begin{array}{l}\text { a) ¡Muy bien! el auto está andando. Hagamos que } \\
\text { se estacione frente a la casa. } \\
\text { b) La mamá quiere ir a comprar. Vamos en el auto } \\
\text { al supermercado. }\end{array}$ \\
\hline Presentación del juguete e invitación a jugar & 5.- Mira este auto, juguemos. & $\begin{array}{l}\text { a) Vamos a pasear en el auto. Qué auto escoges, } \\
\text { por dónde avanzará. } \\
\text { b) Hagámoslos avanzar por la pista de carrera. } \\
\text { c) El chofer está cansado. Necesita detenerse a } \\
\text { dormir. Llevémoslo a su casa. } \\
\text { d) Se pinchó una rueda. ¿Cómo la arreglamos? }\end{array}$ \\
\hline
\end{tabular}

Fuente: Elaboración propia. 
La intencionalidad de generar secuencias en complejidad creciente, ajustadas a los requerimientos y perfil de fortalezas y debilidades de cada niño, permite situar las sesiones sistemáticas de juego, incorporando a la familia y a los profesionales, en los contextos naturales en que se desenvuelven los niños. Esta propuesta desarrollada en forma sistemática permitiría a los niños pasar de conductas funcionales a conductas más complejas en el plano del juego, de la interacción social, de la comunicación y del desarrollo afectivo.

\section{A modo de conclusión}

El juego es una habilidad que forma parte de la vida del ser humano, enriquece su experiencia y permite otorgar sentido y significado a las actividades cotidianas. Éste involucra ámbitos cognitivos, lingüísticos sociales y culturales, convirtiéndose en una experiencia natural que fluye a lo largo del desarrollo, no obstante en el caso de niños y niñas con rezago o que presentan dificultades en algún área específica o discapacidad, este aspecto también se ve interferido. La investigación sugiere que los efectos de una intervención sistemática en niños con discapacidad, participando de experiencias de juegos de imitación y de ficción o de juego simbólico, permite que los niños adquieran mantengan y generalicen las conductas de juego hacia otros contextos (Barton y Wolery, 2010; Barton, 2015). Una propuesta integral que permita a niños y niñas interactuar en forma sistemática con sus padres y sus familias en sesiones de juego, permitiría a partir de un apoyo basado en aspectos socioemocionales y afectivos, transitar desde un juego dirigido y con altos niveles de acompañamiento hacia el logro de otros dominios del desarrollo, incluyendo un juego espontáneo con formas de comunicación más complejas y respetando en todo momento las ideas, necesidades y requerimientos que propongan los niños en este proceso.

Iniciar este acompañamiento en edades tempranas, permitiría prevenir dificultades o retrasos en el desarrollo y evitar que se acentúen conductas de aislamiento en niños con discapacidad. Las secuencias progresivas con graduación del acompañamiento desde moderadas a pequeñas ayudas permitirían generar oportunidades de comunicación, de socialización con pares y de interacción en actividades lúdicas con otros niños. De esta forma se sugiere la inclusión en edades tempranas, tomando como base las posibilidades que brinda el juego para favorecer en forma intencionada el mayor despliegue emocional y social en niños con desarrollo típico y especialmente en aquellos que requieren de ayudas específicas para avanzar en ese desarrollo.

\section{Referencias}

Ainscow, M., Dyson, A., Goldrick, S. y West, M. (2013). Promoting equity in education. Revista de Investigación en Educación, 11 (3), 32-43.

Allen, K. y Cowdery, G. (2015). The exceptional child. Inclusion in early childhood education. Nueva York, NY: Cengage Learning

Aranda, R. (2011). Atención temprana en educación infantil. Madrid: Wolters Kluwer.

Barrachina, L., Aguado, G., Cardona, M. y Sanz-Torrent, M. (2015). El trastorno específico del lenguaje. Barcelona: Editorial UOC. 
Barton, E. (2015). Teaching generalized pretend play and related behaviors to young children with disabilities. $\quad$ Exceptional Children, $\quad 81(4), \quad 489-506$. https://doi.org/10.1177/0014402914563694

Barton, E. y Wolery, M. (2010). Training teachers to promote pretend play in young children with disabilities. Exceptional Children, $\quad$ 87(1), https://doi.org/10.1177/001440291007700104

Bay Area Early Childhood Funders. (2007). The value of play for early learning. Recuperado de http://earlychildhoodfunders.org/

Booth, T. y Ainscow, M. (2015). Index for Inclusion: Developing learning and participation in schools. Bristol: CSIE.

Booth, T. y Ainscow. M. (2011). Index for inclusion. Developing leaning and participation in schools. Manchester: CSIE.

Booth, T., Ainscow, M. y Kingston, D. (2007). Index para la inclusión: Desarrollo del juego, el aprendizaje y la participación en educación infantil. Bristol: CSIE.

Booth, T., Simón, C., Sandoval., Echeita, G. y Muñoz, Y. (2015). Guía para la Educación Inclusiva. Promoviendo el Aprendizaje y la Participación en las Escuelas: Nueva Edición Revisada y Ampliada. REICE. Revista Iberoamericana sobre Calidad, Eficacia y Cambio en Educación, 13(3), 5-19.

Breinbauer, C. (2006). Fortaleciendo el desarrollo de niños con necesidades especiales: Introducción al modelo DIR y la terapia floortime o juego circular. Transiciones. Revista de la Asociación Peruana de Terapia Psiconalíticade Niños y Adolescentes, 11, 1-12.

Bronfenbrenner, U. (1987). La ecología del desarrollo humano. Experimentos en entornos naturales y diseñados. Barcelona: Paidós.

Caillois, R. (1938). Teoría de los juegos. Barcelona: Seix Barral.

Caillois, R. (1986). Los juegos y los hombres. Ciudad de México: Fondo de Cultura Económica.

Clemente, R. (2006). Desarrollo del lenguaje. Barcelona. Octaedro.

Córdoba, E., Lara, F. y García, A. (2017). El juego como estrategia lúdica para la educación inclusiva del buen vivir. Ensayos. Revista de la Facultad de Educación de Albacete, 32(1) 81-92.

DEC/NAEYC. (2009). Early childhood inclusion. Chapel Hill, NC: The University of North Carolina.

Echeita, G. (2013). Definir la inclusión para llevarla a la práctica. En la Calle: Revista sobre Situaciones de Riesgo Social, 26, 11-15.

Echeita, G. y Navarro, A. (2014). Educación inclusiva y desarrollo sostenible. Una llamada urgente a pensarlas juntas. Edetania, 46, 141-161.

Espinoza, M. (1999). El estudio observacional del juego en los preescolares. En T. Anguera (Coord.). Observación de la escuela (pp. 281-288). Barcelona: Ediciones UB.

Fromberg, D. (2002). Play and meaning in early chilhood education. Boston, MA: Allyn \& Bacon.

García, F. S. (2014). Atención temprana, enfoque centrado en la familia. En AELFA. (Ed.), Actas del XXIX Congreso AELFA. Logopedia: evolución, transformación y futuro (pp. 286-302). Madrid: AELFA.

Garfinkle, A. (2004). Assessing play skills. En M. Mc Lean, M. Wolery y D. Bailey (Eds.), Assessing infants and preeschoolers with special needs (pp. 451-516). Upple Sadder River, NJ: Pearson.

Garvey, C. (1985). El juego infantil. Madrid: Morata. 
Giaconi, C., Nahuelhuala, P. y Pedrero, V. (2017). Participación de niños y adolescentes en situación de discapacidad: Un ámbito a ser estudiado y evaluado. Revista Chilena de Pediatría, 88(1), 184-185.

Greenspan, S. y Wieder, S. (1999). A functional developmental approach to autistic spectrum disorders. JASH, 24(3), 147-161.

Hughes, F. (1991). Children, play and development. Boston, MA: Allyn \& Bacon.

Huizinga, J. (1972). Homo ludens. Ciudad de México: Fondo de Cultura Económica.

Martínez-Priego, C., Anaya-Hamue, M. E. y Salgado, D. (2014). Desarrollo de la personalidad y virtudes sociales: Relaciones en el contexto educativo familiar. Educación y Educadores, $17(3)$, 447-467. https://doi.org/10.5294/edu.2014.17.3.3

Mc William, R. (2016). Metanoia en atención temprana: Transformación a un enfoque centrado en la familia. Revista Latinoamericana de Educación Inclusiva, 10(1) 133-153. https://doi.org/10.4067/S0718-73782016000100008

Meyer, A., Rose, D. H. y Gordon, D. (2014). Universal design for learning: Theory and practice. Wakefield, MA: CAST Professional Publishing.

OMS. (2001). Clasificación Internacional del funcionamiento, de la discapacidad y de la salud. Madrid: Imserso.

OMS. (2013). El desarrollo del niño en la primera infancia y la discapacidad. Un documento de debate. Malta: OMS.

OMS. (2017). Diez datos acerca del desarrollo de la primera infancia como determinante social de la salud. Recuperado http://www.who.int/maternal_child_adolescent/topics/child/development/10facts/es/

ONU. (1959). Declaración de los derechos del niño. Recuperado de https://www.oas.org/dil/esp/Declaraci\%C3\%B3n\%20de\%20los\%20Derechos\%20del\%20 Ni\%C3\%B10\%20Republica\%20Dominicana.pdf

ONU. (1989). Convención sobre los derechos del niño. Recuperado de http://www.ohchr.org/SP/ProfessionalInterest/Pages/CRC.aspx

Owens, R. (2016). Language development an introduction. Boston, MA: Pearson.

Peredo, R. (2016). Comprendiendo la discapacidad intelectual: Datos, criterios y reflexiones. Reflexiones en Psicología, 15, 101-122.

Perpiñan, S. (2003). Generando entornos competentes. Padres, educadores, profesionales de AT: Un equipo de estimulación. Revista de Atención Temprana, 6(1) 11-17.

Sahlberg, P. (2013). El cambio educativo en Finlandia. ¿Qué puede aprender el mundo? Buenos Aires: Paidós.

Sherratt, D. y Peter, M. (2002). Developing play and drama in children with autistic spectrum disorders. Recuperado de https://www.amazon.co.uk/Developing-Children-Autistic-SpectrumDisorders/dp/ 1853466972

Smith, P. y Vollstedt, L. (1985). On defining play: An empirical study of the relationship between play and various play criteria. Child Development, 56(4) 1042-1050. https://doi.org/10.2307/1130114

UNESCO. (2009). Directrices sobre políticas de educación inclusiva. París: Organización de las Naciones Unidas para la Educación, la Ciencia et la Cultura. 
UNESCO. Acuerdo de 2014$)$ Mascate. Recuperado de: http://www.unesco.org/fileadmin/MULTIMEDIA/FIELD/Santiago/pdf/MuscatAgreement-ESP.pdf

UNESCO. (2015). Declaración de Incheon. Recuperado de: https://es.unesco.org/world-educationforum-2015/about-forum/declaracion-de-incheon

UNESCO. (2016). Training tools for curriculum development: A resource pack supporting Inclusive education. Ginebra: International Bureau of Education-Unesco.

Whitebread, D., Basilio, M., Kuvalja, M. y Verma, M. (2012). The importance of play. Bruselas: Toy Industries of Europe.

Wieder, S. y Greenspan, S. (2005). Can children with autism master the core deficits and become empathetic, creative, and reflective? The Journal of Developmental and Learning Disorders, 9, $1-29$.

Winnicott, D. (2012) Realidad y juego. Buenos Aires: Gedisa.

Zych, I., Ortega-Ruiz, R. y Sibaja, S. (2016). Children's play and affective development affect, school adjustment and learning in preschoolers. Infancia y aprendizaje, 39, 380-400. https://doi.org/10.1080/02103702.2016.1138718

\section{Breve CV de las autoras}

\section{Francisca Cáceres Zúñiga}

Profesora de Educación Especial y Diferenciada. Pontificia Universidad Católica de Chile. Doctora en Ciencias Humanas, Mención Discurso y Cultura. Universidad Austral de Chile. Magíster en Política y Gestión Educacional. Universidad de Talca. Profesor Instructor. Departamento de Diversidad e Inclusividad Educativa. Facultad de Ciencias de la Educación. Universidad Católica del Maule. Miembro del Centro de Investigación en Educación para la Justicia Social, CIEJUS. Líneas de investigación vinculadas a: Juego, Comunicación y Lenguaje, Inclusión Educativa. ORCID ID: 0000-0001-7280-9616.Email: mfcaceres@ucm.cl

\section{Maribel Granada Azcárraga}

Profesora de Educación Diferencial con Mención en Aucdición y Lenguaje. Universidad Metropolitana de Ciencias de la Educación. Doctora en Filosofía y Ciencias de la Educación, Universidad Complutense de Madrid. Profesor Adjunto, Departamento de Diversidad e Inclusividad Educativa. Facultad de Ciencias de la Educación. Universidad Católica del Maule. Miembro del Centro de Investigación en Educación para la Justicia Social, CIEJUS. Líneas de investigación vinculadas a: Comunicación y Lenguaje e Inclusión Educativa. ORCID ID: OOOOoo03-1123-3566. Email:mgranada@ucm.cl

\section{María Pomés Correa}

Profesora de Educación Especial y Diferenciada. Pontificia Universidad Católica de Chile. PhD en Filosofía, Educación Especial, Intervención Temprana. Universidad de Oregon - EE. UU. Profesor Adjunto. Departamento de Diversidad e Inclusividad Educativa. Facultad de Ciencias de la Educación. Universidad Católica del Maule. Miembro del Centro de Investigación en Educación para la Justicia Social, CIEJUS. Líneas de investigación vinculadas a: Atención Temprana, Prevención y evaluación de las dificultades del Aprendizaje, Comunicación y Lenguaje, Inclusión Educativa. ORCID ID: 0000-0003-2220-7451. Email: mppomes@ucm.cl 
Revista Latinoamericana de Educación Inclusiva 